\title{
Diferencias en los niveles de actividad física, grado de adherencia a la dieta mediterránea y autoconcepto físico en adolescentes en función del sexo \\ Gender differences in physical activity levels, degree of adherence to the Mediterranean diet, and physical self-concept in adolescents \\ Alejandro Tapia López \\ Universidad de Alicante
}

\begin{abstract}
Resumen. El propósito de este trabajo es conocer los niveles de actividad física, el grado de adherencia a la dieta mediterránea y el autoconcepto físico en una muestra de estudiantes de $3^{\circ}$ y $4^{\circ}$ de la ESO de Benidorm (España). Además, se pretende conocer si existen diferencias en función del sexo en las distintas variables estudiadas. El número de participantes es de 189, siendo el $47.62 \%$ chicos ( $n=$ 90) y el $52.38 \%$ chicas $(n=99)$, con edades comprendidas entre los 14 y 17 años $(M=14.76, D T=0.75)$. Se han utilizado diversos instrumentos como el cuestionario sobre la práctica de actividad física (elaboración propia), el cuestionario KIDMED y el cuestionario de autoconcepto físico (CAF). Los resultados de la investigación revelan la necesidad urgente de actuación para la mejora de la adherencia a la actividad física y un adecuado patrón alimentario (dieta mediterránea), observándose diferencias en función del sexo en los niveles de actividad física pero no en el grado de adherencia a la dieta mediterránea. Asimismo, se observan peores puntuaciones en todas las escalas del autoconcepto físico en las chicas, a excepción del atractivo físico. Resulta necesaria una actuación para fomentar hábitos saludables en adolescentes que haga partícipe a las familias, los centros educativos y las administraciones públicas.
\end{abstract}

Palabras clave. Actividad física; Dieta mediterránea; Autoconcepto físico; Adolescentes; España.

Abstract. The purpose of this project is to assess physical activity levels, degree of adherence to the Mediterranean diet, and physical self-concept in a sample of students from 3rd and 4th grade of ESO (Compulsory Secondary Education) in Benidorm (Spain). Furthermore, it aims to see if there are differences by gender in the studied variables. The sample is composed by 189 students, $47.62 \%$ of which are boys ( $n=90)$, and $52.38 \%$ are girls $(n=99)$. Their ages vary from 14 to 17 years old $(M=14.76, D T=0.75)$. Different tools, such as an ad-hoc questionnaire about their physical activity, the KIDMED questionnaire, and the physical self-concept questionnaire (CAF), were employed. The results of this research reveal an urgent necessity for action to improve adherence to physical activity and to an adequate diet (Mediterranean diet); the statistical differences between boys and girls are significant in physical activity levels, but not in the degree of adherence to the Mediterranean diet. Likewise, worse results are observed in all measurements of physical self-concept in girls, with the exception of physical attractiveness. In order to promote healthy habits in adolescents, it appears necessary to carry out an action plan that involves families, schools, and public administrations.

Keywords. Physical activity; Mediterranean diet; Physical self-concept; Adolescents; Spain.

\section{Introducción}

La promoción de estilos de vida activos en la adolescencia resulta importante, ya que al ser una etapa crítica en el desarrollo de una persona y determinante para su evolución futura, puede influir notablemente en la vida adulta (Blakemore \& Mills, 2014). La práctica de una actividad física regular, estable y moderada ayuda a mejorar tanto la salud física como psicológica y social (Chen \& Lee, 2013; Moreno, Moreno \& Cervelló, 2009). Existe suficiente evidencia científica para afirmar que la práctica de actividad física contribuye a la prevención primaria y secundaria de numerosas enfermedades crónicas y se asocia a un menor riesgo de muerte prematura (Warburton, Nicol \& Bredin, 2006). Por desgracia, los niveles de actividad física realizada por los adolescentes españoles son claramente inferiores a las recomendaciones (Ramos, Jiménez-Iglesias, Rivera \& Moreno, 2016). La asignatura de Educación Física, dado su carácter obligatorio en el currículum educativo, es para muchos adolescentes el único lugar donde realizan actividad física regularmente (Sevil, Abós, Generelo, Aibar \& García-González, 2016). Por tanto, es una oportunidad que no hay que desaprovechar para introducir al alumno en la práctica de actividad física con la intención de que mantenga esta conducta en la edad adulta. El papel

Fecha recepción: 12-08-18. Fecha de aceptación: 28-01-19 Alejandro Tapia López

tapialopezalejandro@gmail.com del docente resulta fundamental en la creación de un adecuado ambiente, clima motivacional y experiencias satisfactorias para el logro de dicho fin (Aibar et al, 2015; Gutiérrez, 2014; Moreno \& Cervelló, 2004).

Sin embargo, la adquisición de conductas saludables y su mantenimiento en el tiempo no deben centrarse únicamente en la práctica de actividad física, sino también en la adherencia a adecuados hábitos alimentarios. Diversos estudios epidemiológicos han constatado un incremento en la longevidad y reducción en la morbilidad en países mediterráneos respecto a Estados Unidos o el norte de Europa (Keys et al., 1986; Tunstall-Pedoe et al., 1999), atribuyendo dichos beneficios para la salud al patrón dietético (Menotti et al., 1999). A pesar de las variaciones en los componentes de la dieta mediterránea (DM) tanto dentro de un país como entre distintos países, existe un patrón común: un consumo elevado de alimentos de origen vegetal como verduras, hortalizas, frutas, legumbres, frutos secos y cereales, con el aceite de oliva como principal fuente de grasa; un consumo moderado de pescado, productos lácteos, aves y huevos; y un bajo consumo en frecuencia y cantidad de carnes rojas y embutidos (Buckland, Bach \& Serra, 2008; Willet et al., 1995). A pesar de que los resultados de los distintos estudios que investigan la relación entre la DM y el sobrepeso u obesidad son controvertidos debido a las diferencias metodológicas aplicadas, todo parece indicar que la DM es un factor protector sobre la ganancia de peso (Buckland et al., 2008). Estudios como el de Calatayud, Calatayud \& Gallego (2011) han 
comprobado los efectos de una DM sin restricción calórica y sin intervención específica sobre práctica física en niños y adolescentes con sobrepeso y obesidad, obteniendo un descenso significativo de la masa grasa corporal. Sin embargo, los mejores resultados se obtienen cuando se combina una dieta controlada con la práctica de ejercicio (Aguilar et al., 2014; Bonfanti, Fernández, Gómez-Delgado \& Pérez-Jiménez, 2014; Foster-Schubert et al., 2012; Frimel, Sinacore \& Villareal, 2008; González, Hernández, Pozo \& García, 2011; Stephens, Cobiac \& Veerman, 2014). De este modo, combinando DM con programas de actividad física, autores como Esposito et al. (2003) han logrado en sus investigaciones importantes pérdidas de peso.

Por otro lado, son numerosas las variables que pueden repercutir en el bienestar adolescente. Durante la adolescencia, el autoconcepto es uno de los elementos más importantes para un desarrollo equilibrado (Esnaola, Goñi \& Madariaga, 2008) y constituye un gran reto debido a los cambios cognitivos, físicos y sociales que se producen (Esnaola, 2005b). El autoconcepto se refiere a la percepción que tienen las personas de sí mismas y se genera a partir de las experiencias e interpretaciones que se hacen del contexto ambiental (Esnaola, 2005a). Un autoconcepto positivo se relaciona con el correcto funcionamiento personal y social, estando implicado en la relación con el entorno, en la adquisición de conductas saludables y el rendimiento en ámbitos como el académico, social o deportivo (Guillén \& Ramírez, 2011; Slutzky \& Simpkins, 2009). Por el contrario, si se tiene un autoconcepto negativo es más fácil caer en depresión, drogas o alcohol (Esnaola, 2005b). En la actualidad, el modelo de autoconcepto que predomina es de naturaleza multidimensional e incluye las dimensiones física, emocional, familiar, académica y social (Esnaola, Rodríguez \& Goñi, 2011; Shavelson, Hubner \& Stanton, 1976). El autoconcepto físico lo componen, a su vez, cuatro subdominios que son: habilidad deportiva, atractivo físico, condición física y fuerza física (Fox \& Corbin, 1989; Shavelson et al., 1976).

\section{Objetivos e hipótesis}

El presente estudio pretende conocer los niveles de actividad física, el grado de adherencia a la dieta mediterránea y el autoconcepto físico de una muestra de estudiantes de $3^{\circ} \mathrm{y}$ $4^{\circ}$ de la ESO. Asimismo, se pretende verificar si existen diferencias en función del sexo en las distintas variables estudiadas. Se plantean las siguientes hipótesis:

- Los niveles de actividad física realizada por la muestra de adolescentes es inferior a las recomendaciones.

- Los chicos presentan mayores niveles de actividad que las chicas.

- La adherencia a la dieta mediterránea es insuficiente y se deben realizar programas educativos para la mejora del patrón alimentario.

- No existen diferencias en la adherencia a la dieta mediterránea en función del sexo.

- Las chicas obtienen peores puntuaciones respecto a los chicos en todos los subdominios del autoconcepto físico.

\section{Método}

\section{Participantes}

En el estudio participaron 189 adolescentes (90 chicos y 99 chicas) con una edad comprendida entre los 14 y los 17 años $(M=14.76, D T=0.75)$ pertenecientes a $3^{\circ}$ y $4^{\circ}$ de la ESO del Instituto de Educación Secundaria L’Almadrava y del Instituto de Educación Secundaria Mediterrània (Benidorm, España). Los criterios de inclusión fueron tener rellenados en cada cuestionario los datos de referencia (sexo, edad y centro educativo), no dejar preguntas sin responder y no contestar dos veces la misma pregunta. Para la realización del presente estudio, se solicitó permiso a los centros educativos, así como el consentimiento informado de los padres o tutores legales. Asimismo, la participación fue totalmente voluntaria, de forma que independientemente de tener el consentimiento de los padres, solo se entregaron cuestionarios a aquellos alumnos que quisieron formar parte de la muestra.

\section{Instrumentos}

a. Cuestionario sobre la práctica de actividad física. Se trata de un cuestionario de elaboración propia en el que se preguntó a los estudiantes si realizaban actividad física con dos posibilidades de respuesta «sí/no». Antes de cumplimentar dicho cuestionario se les hizo saber que no era necesario estar federado en ningún deporte y que se entiende por práctica de actividad física la realización de cualquier actividad de tipo físico fuera del horario escolar en la que se produzca un aumento de la frecuencia cardíaca junto a la aparición de sudor (bailar, montar en bicicleta, nadar, correr, etc.). En caso de responder positivamente a la pregunta, debían responder dos preguntas más «¿Cuántos días por semana?» y «¿Cuánto tiempo practicas los días que haces actividad física?», existiendo tres posibilidades de respuesta en ambos casos: «1/2-3/4 o más» $\mathrm{y}$ «menos de 30 minutos/ entre 30 y 60 minutos/más de 60 minutos», respectivamente. De esta forma se categorizó a los adolescentes en tres niveles: un nivel para los que no realizan ningún tipo de actividad física o la actividad realizada es ínfima (nivel 0/sedentarios), un nivel para aquellos que realizan actividad física por debajo de las recomendaciones (nivel 1/poco activos) y un último nivel para los que cumplen las recomendaciones (nivel 2/ activos). La categorización de los alumnos en los distintos niveles (tabla 1) se realizó en base a las recomendaciones de actividad física en adolescentes, en las que se aconseja al menos 1 hora diaria de actividad física moderada o vigorosa (Kahlmeier et al., 2015; OMS, 2010; Physical Activity Guidelines Advisory Committee, 2018). Estas recomendaciones incluyen toda actividad física realizada durante el día, lo que incluye aquella realizada durante el horario escolar. Sin embargo, los participantes de este estudio tan solo indicaron la actividad física realizada fuera del horario escolar, por lo que es probable que el tiempo dedicado a la misma sea ligeramente superior gracias a la contribución de la asignatura de Educación Física y, en algunos casos, al empleo activo del recreo por parte del alumnado. Por este motivo, se delimitó como activos tanto a aquellos que señalaron 4 o más días por semana de actividad física con una duración de más de 60 minutos como a aquellos que señalaron 4 o más días por 
semana de actividad física con una duración de entre 30 y 60 minutos.

b. Cuestionario KIDMED (Serra-Majem et al., 2004). Este instrumento valora la adherencia a la dieta mediterránea mediante 16 preguntas que deben responderse de manera afirmativa/negativa (sí/no) de las cuales 12 puntúan positivamente, en caso de responderse afirmativamente al representar un aspecto positivo en relación con la dieta mediterránea, y 4 puntúan negativamente, en caso de responderse afirmativamente al representar un aspecto negativo en relación con la dieta mediterránea. Las respuestas negativas no puntúan. De esta forma, la puntuación obtenida en dicho test puede oscilar entre -4 y 12 puntos. Los autores sugieren que una puntuación igual o inferior a 3 puntos supone una dieta de muy baja calidad, una puntuación entre 4 y 7 puntos refleja la necesidad de mejorar el patrón alimentario para adecuarlo a la dieta mediterránea y, una puntuación entre 8 y 12 puntos permite afirmar que existe una alta adherencia a la dieta mediterránea.

c. Cuestionario de autoconcepto físico/CAF (Goñi, Ruiz de Azúa \& Liberal, 2004). El instrumento lo componen 36 ítems distribuidos en cuatro escalas específicas de autoconcepto físico (habilidad deportiva, condición física, atractivo físico y fuerza) y dos escalas generales (autoconcepto físico general y autoconcepto general). De los 36 ítems (seis por escalas), 20 están redactados de manera directa, por lo que se puntúan de 1 a 5 siendo 1 falso y 5 verdadero, y 16 de manera indirecta, por lo que se puntúan de 5 a 1 siendo 5 falso y 1 verdadero. Los análisis de fiabilidad originales medidos a través de Alfa de Cronbach fueron $\alpha=.80$ (habilidad deportiva), $\alpha=.84$ (condición física), $\alpha=$ .88 (atractivo físico), $\alpha=.83$ (fuerza), $\alpha=.88$ (autoconcepto físico general) y $\alpha=.79$ (autoconcepto general).

\section{Procedimiento}

Para la realización de este trabajo se contactó con los centros de educación solicitando el permiso pertinente, así como el consentimiento informado de los padres o tutores legales de los alumnos para que pudieran participar en la investigación. La cumplimentación de los cuestionarios se realizó entre diciembre y febrero del curso 2017/2018 durante la hora de Tutoría, estando el investigador presente en todo momento y siendo el responsable de dar las instrucciones para la cumplimentación de los distintos instrumentos y resolver las dudas que pudieran surgir tanto en la comprensión de los ítems como de las instrucciones establecidas al inicio de la administración de los cuestionarios. Asimismo, los alumnos se repartieron por el aula dejando separación entre ellos para mantener la confidencialidad en las respuestas y se prohibió la posibilidad de comentar las respuestas entre ellos. Un aspecto a destacar es que se recalcó en todo momento la voluntariedad y el anonimato en la participación, motivo por el cuál se solicitó máxima sinceridad en la cumplimentación de los distintos instrumentos.

\section{Análisis de datos}

Se ha realizado un análisis descriptivo de las distintas variables presentes en el estudio. Se efectuaron contrastes de normalidad a través de la prueba de Kolmogorov-Smirnov, motivo por el cual se decidió aplicar pruebas no paramétricas.
Se aplicó la U de Mann-Whitney para las variables numéricas y la prueba F exacta de Fisher y la Chi-cuadrado para las variables categóricas. Para el análisis de consistencia interna del CAF se utilizó el coeficiente alfa de Cronbach. Se consideraron como estadísticamente significativos los resultados con un $p$ valor $<.05$. El programa estadístico utilizado es el SPSS en su versión 23.0.0.0 para Windows (IBM ${ }^{\circledR}$ SPSS ${ }^{\circledR}$ STATISTICS).

\section{Resultados}

\section{Actividad física}

Los datos muestran niveles de actividad física inferiores a las recomendaciones (tabla 2). En general, el 33.3\% de la muestra es sedentaria (nivel 0), el 38.6\% realiza actividad física por debajo de las recomendaciones (nivel 1), y tan solo el 28\% cumplen con las recomendaciones (nivel 2). No existen diferencias significativas en función del sexo en el nivel $0(p=.067)$ y nivel $1(p=.179)$, pero sí en el nivel $2(p=.001)$ a favor de los chicos.

\section{Adherencia a la dieta mediterránea}

Los resultados del cuestionario de adherencia a la dieta mediterránea son alarmantes, ya que tan solo el 19.6\% de los adolescentes tiene un adecuado patrón alimentario mientras que el $80.4 \%$ necesita acometer cambios en su dieta para mejorar al patrón alimentario y adecuarlo a la dieta mediterránea (tabla 3). La prueba U de Mann-Whitney reveló que no existen diferencias en función del sexo $(p=.976)$.

\section{Autoconcepto físico}

El análisis de consistencia interna del CAF, realizado a través del coeficiente alfa de Cronbach, reveló los siguientes valores: $\alpha=.86$ (habilidad deportiva), $\alpha=.87$ (condición física), $\alpha=.85$ (atractivo físico), $\alpha=.83$ (fuerza), $\alpha=.87$

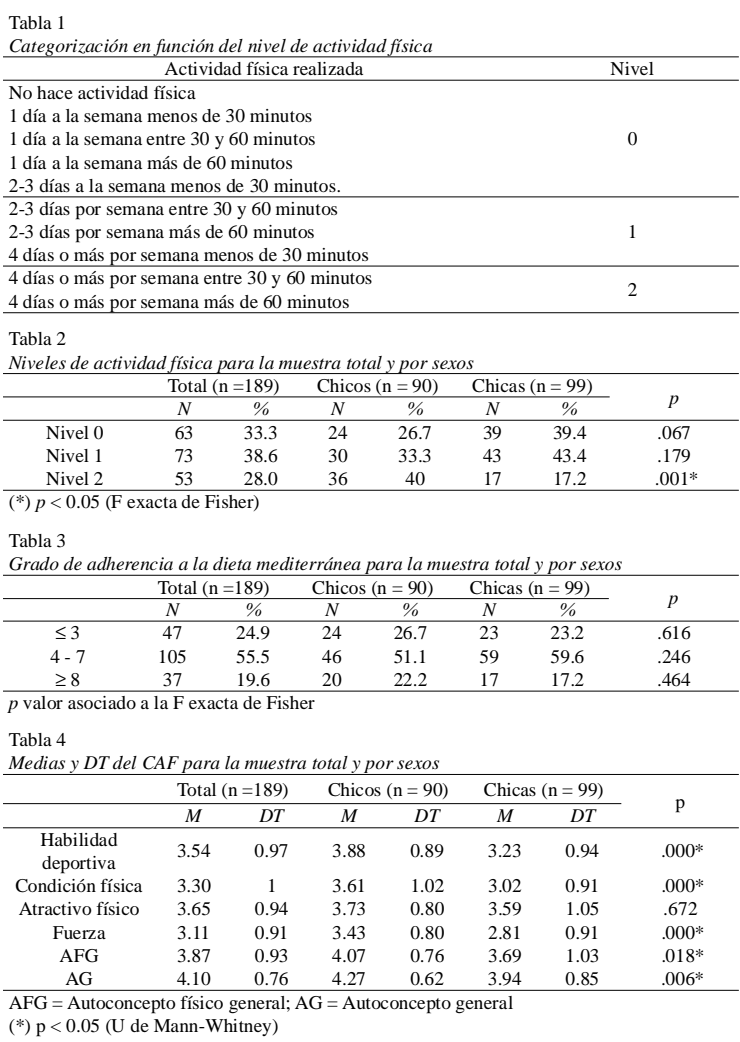


(autoconcepto físico general) y $\alpha=.75$ (autoconcepto general). En relación a las medias obtenidas en el cuestionario de autoconcepto físico, tanto para la globalidad de la muestra como para ambos sexos se observó mayores puntuaciones en las escalas generales que en las específicas (tabla 4). Sin embargo, en las específicas, los chicos obtienen la mayor puntuación en habilidad deportiva mientras que las chicas en atractivo físico. En cualquier caso, las puntuaciones de los chicos son significativamente superiores en todas las escalas respecto a las chicas a excepción del atractivo físico $(p=.672)$.

\section{Discusión}

Los resultados de la presente investigación reflejan que un porcentaje alto de alumnos (71.9\%) muestran una actitud sedentaria o realizan actividad física por debajo de las recomendaciones (nivel 0 y 1). En cualquier caso, solo el $28 \%$ de los adolescentes (nivel 2) cumplen las recomendaciones sobre práctica física. Estos resultados concuerdan con otras investigaciones en las que se alerta de un alto nivel de inactividad entre la población adolescente (Beltrán-Carrillo, DevísDevís \& Peiró-Velert, 2012; Oviedo et al., 2013; Ramos et al., 2016). Numerosos estudios afirman que existe una mayor practica física en los chicos respecto a las chicas (BeltránCarrillo et al., 2012; Cuervo, Cachón, González \& Zagalaz, 2017; Oviedo et al., 2013; Ramos et al., 2016). Cabe destacar la investigación realizada por Beltrán et al. (2017) en la que se manifiestan diferencias según género en el tiempo empleado en actividad sedentaria y actividad física en diferentes segmentos horarios del día, y en la que se hace hincapié en la necesidad de promover la actividad física en las chicas. Los estudios mencionados anteriormente van en línea con los resultados obtenidos en la presente investigación, en la que se observan diferencias significativas respecto a los que cumplen las recomendaciones a favor de los chicos ( $p=$ .001). En cualquier caso, resulta preocupante que el $60 \%$ de los chicos y el $82.8 \%$ de las chicas no cumplan con las recomendaciones de actividad física en adolescentes.

Los datos obtenidos justificarían la necesidad de crear programas de actividad física y redes de colaboración entre el entorno más cercano de los jóvenes (familias, centros educativos y administración local). Estos programas deben incluir una amplia oferta tanto de actividades dirigidas, preferiblemente con un fin lúdico, como de espacios en los que practicar actividad física de forma libre (carriles bici, parques de patinaje, zonas de juego, etc.). Puede resultar interesante la promoción del transporte activo en el trayecto al centro educativo con el fin de fomentar un estilo de vida activo (Beltrán et al., 2017). Estevan, Queralt \& Molina-García (2018) resaltan la importancia de los sistemas de bicicletas compartidas y de adecuadas infraestructuras para el uso de la bicicleta como medio de transporte al centro educativo en adolescentes. Adicionalmente, puede resultar interesante la implementación de programas de actividad físico-deportiva enfocados al empleo activo del recreo (Lamoneda \& Huertas, 2017). Se debe prestar especial atención al colectivo femenino de forma que el abanico de posibilidades para la realización de actividad física, entre las que se incluye el currículo de Educación Física, también sea atractivo para ellas. Estudios como el de Lamoneda \& Huertas (2017) reflejan que pueden producirse diferencias significativas entre chicos y chicas en la participación a programas de promoción de la actividad física en función del contenido, del tipo de interacción motriz y de la instalación utilizada.

Respecto a la adherencia a la DM, resulta difícil realizar comparaciones entre los distintos estudios que aplican el KIDMED en jóvenes debido a las diferencias metodológicas aplicadas, principalmente, en la selección de la muestra. La media de los valores de la puntuación total del cuestionario de adherencia a la DM obtenida en el presente estudio (5.4 \pm 2.5) es similar a la obtenida por Gussinyer et al. (2008) antes de su intervención, aunque en este último estudio los participantes son niños con sobrepeso u obesidad de entre 6 y 12 años visitados en la Unidad de Endocrinología Pediátrica del Hospital Materno-infantil Vall d'Hebron (Barcelona). Sin embargo, los resultados obtenidos en el Instituto de Educación Secundaria L'Almadrava y el Instituto de Educación Secundaria Mediterrània (Benidorm, España) respecto a otros estudios realizados en centros educativos de España y con participantes de edad similar son inferiores (Grao-Cruces et al., 2013; Grao-Cruces, Nuviala, Fernández-Martínez \& Martínez-López, 2015) o muy inferiores (Ayechu \& Durá, 2010; Mariscal-Arcas et al., 2009), lo que revela la necesidad de una actuación urgente. Mariscal-Arcas et al. (2009) aprecia diferencias significativas en el KIDMED en función del sexo en niños menores de 10 años, sin embargo, estas diferencias no se dan en adolescentes (Ayechu \& Durá, 2010; Grao-Cruces et al., 2013; Mariscal-Arcas et al., 2009). En un estudio reciente de Rosa et al. (2018) con una muestra de 520 estudiantes de entre 8 y 17 años $(12.81 \pm 2.99)$ sugieren que la edad influye en la DM y, al igual que las investigaciones citadas anteriormente, tampoco obtienen diferencias en función del sexo. Resultados semejantes se han observado en este estudio al no existir diferencias significativas en función del sexo $(p=.976)$.

La familia tiene una influencia notable sobre los hábitos de los jóvenes pudiendo presentarse incluso como una barrera para la actividad física y una dieta saludable (Aguilar et al., 2014), por lo que cada vez más estudios incluyen la participación de las familias en sus intervenciones (Bibiloni et al., 2017; Calatayud et al., 2011; García-Reyna, Gussinyer \& Carrascosa, 2007; Gussinyer et al., 2008). Diversos estudios han relacionado el nivel de actividad física de padres y madres con el de sus hijos, existiendo una mayor probabilidad de realizar actividad física en éstos si sus padres y madres son practicantes (Marques, González, Martins, FernándezVillarino \& Carreiro, 2017; Piéron \& Ruiz-Juan, 2013). Por añadidura, los adolescentes que atribuyen a sus progenitores unas valoraciones más elevadas en el ejercicio de una parentalidad transformacional presentan un mayor autoconcepto físico (De la Torre, Ruiz-Ariza, López-Serrano \& Martínez-López, 2018), así como una mayor práctica de actividad física y hábitos alimentarios saludables (Morton, Wilson, Perlmutter \& Beauchamp, 2012). A tenor de los resultados obtenidos en el KIDMED y en el cuestionario sobre práctica de actividad física, y a modo de propuesta, sería interesante realizar jornadas informativas y de concienciación sobre la importancia de seguir hábitos saludables en los que participasen tanto los jóvenes como sus familias. Se deben 
explorar vías para que el entorno del adolescente se involucre y sea un modelo a seguir. Además, puede resultar de interés realizar intervenciones familiares con el propósito de incrementar los comportamientos transformacionales.

Por otro lado, son numerosos los estudios que afirman que las mujeres son más críticas con sus cuerpos, se preocupan más por la apariencia física que los hombres y asocian más el atractivo corporal con un mejor autoconcepto físico general (De Gracia, Marcó \& Trujado, 2007; Loland, 1998; Moreno et al., 2009). La imagen corporal es especialmente importante en mujeres jóvenes (Estévez et al., 2015). En cambio, los hombres se basan más en la competición deportiva, la exaltación del ego, la aprobación social o la mejora de la condición física (Moreno \& Cervelló, 2005; Moreno, Cervelló \& Moreno, 2008; Moreno et al., 2009). El índice de masa corporal se ha relacionado con el autoconcepto físico, de forma que las personas más delgadas son las que tienen mejor percepción física (Crocker, Sabiston, Kowalski, Mcdonough \& Kowalski, 2006; Goñi \& Rodríguez, 2007; Fernández-Bustos, González-Martí, Contreras \& Cuevas, 2015). Esta relación es de extrema importancia, ya que un pobre autoconcepto físico puede servir de alerta diagnóstica de trastornos de conducta alimentaria (Goñi \& Rodríguez, 2004). Las mujeres presentan menores puntuaciones en casi todos (Moreno et al., 2009) o en todos (Esnaola \& Revuelta, 2009; Molero, Ortega, Valiente \& Zagalaz, 2010; Reigal \& Videra, 2011; Revuelta, Esnaola \& Goñi, 2013) los subdominios del autoconcepto físico respecto a los hombres. En el presente estudio, las chicas presentan puntuaciones significativamente inferiores en todos los subdominios a excepción del atractivo físico $(p=.672)$. Estos resultados nos permiten ver que para la muestra del estudio ambos sexos se preocupan por igual sobre el atractivo físico, probablemente debido a la creciente presión social y a la importancia que se le otorga durante la adolescencia, aunque los chicos siguen preocupándose más por aspectos relacionados con la práctica deportiva.

Es probable que la actuación sobre cualquiera de las variables estudiadas produzca modificaciones sobre el resto de ellas. Diversos estudios han asociado la práctica de actividad física a un mejor desarrollo del autoconcepto físico en adolescentes (Contreras, Fernández, García, Palou \& Ponseti, 2010; Esnaola \& Revuelta, 2009; Goñi \& Infante, 2010; Molero et al., 2010), siendo este mayor cuanta más actividad física se realiza (Reigal \& Videra, 2011; Revuelta, Esnaola \& Goñi, 2016). Sin embargo, pueden existir diferencias en la influencia de la práctica de actividad física sobre los subdominios del autoconcepto físico y el propio autoconcepto físico en función del género (Reigal \& Videra, 2011). De igual modo, parecen existir asociaciones positivas y significativas entre clima familiar deportivo percibido y autoconcepto físico (Revuelta \& Esnaola, 2011). El autoconcepto físico sirve de puente entre las mejoras acaecidas por la práctica física regular en los distintos subdominios del mismo y el autoconcepto general (Contreras et al., 2010). En un estudio realizado por Moreno, Moreno \& Cervelló (2007) con adolescentes quedó patente que el autoconcepto físico predice la intención de seguir siendo físicamente activo tras la etapa educativa. En dicho estudio, la competencia percibida fue el principal predictor. Pero, más allá de aspectos relacionados con la actividad física, cabe resaltar que las diferentes dimensiones del autoconcepto físico correlacionan significativamente con la percepción de satisfacción vital (Videra-García \& ReigalGarrido, 2013). Asimismo, el autoconcepto físico tiene relación con la conducta de beber y fumar, pudiendo predecir estas conductas mediante la valoración de dicho constructo (Moreno et al., 2009). Usán, Salavera, Murillo \& Merino (2018) afirman, en base a los resultados de su estudio, que una mayor competencia percibida se relaciona con una menor prevalencia de consumo de alcohol, tabaco y cannabis. Los sujetos con mayor autoconcepto también tienen una mejor percepción acerca de la propia alimentación y salud (Rodríguez, Goñi \& Ruiz de Azúa, 2006). Por otro lado, el grado de adherencia a la dieta mediterránea se asocia positivamente con la actividad física (Grao-Cruces et al., 2015; Rosa et al., 2018).

Las principales limitaciones que presenta este estudio son la dificultad para realizar comparaciones con otras investigaciones debido a las diferencias metodológicas aplicadas, la necesidad de ser precavido a la hora de generalizar los resultados para el municipio de Benidorm al ser necesaria una muestra más representativa y mucho más en caso de querer generalizarlos a otras zonas del país. La aplicación de un cuestionario de elaboración propia para medir los niveles de actividad física dificulta aún más este proceso. De igual modo, se afirma en base a otros estudios que las distintas variables están relacionadas, pero no se sabe con exactitud cuál es la dirección de la causalidad.

Por último, parece importante subrayar que es necesaria una actuación para aumentar el número de adolescentes que cumplen con las recomendaciones de actividad física, especialmente en chicas, y tienen un adecuado patrón alimentario. El autoconcepto físico es uno de los elementos que repercuten en el bienestar de los jóvenes y que puede influir en la consecución de los objetivos marcados anteriormente, por lo que debe ser tenido en cuenta además de ser útil para detectar conductas no saludables. Vista la importancia de la percepción de competencia sobre la conducta de práctica física y la intención de seguir físicamente activo tras la etapa educativa, parece prioritario actuar sobre esta dimensión del autoconcepto físico. Si un adolescente se siente competente en las clases de Educación Física es lógico pensar que la probabilidad de adquirir y mantener un estilo de vida activo es mayor. Por consiguiente, si los alumnos logran esa adherencia tan deseada a la práctica regular de actividad física, de forma consecuente mejorarán a su vez otros subdominios como condición física o fuerza. Es imprescindible que los alumnos sientan satisfacción con la práctica, además de evitar las malas experiencias. La adquisición de un estilo de vida activo puede favorecer otras conductas saludables como la adherencia a un adecuado patrón alimentario. Sin embargo, la implementación de programas exige un enfoque multidisciplinar por parte de los centros educativos, y no un esfuerzo único del Departamento de Educación Física. De esta forma, otras asignaturas pueden contribuir a difundir los beneficios que reporta un estilo de vida saludable y los riesgos que supone obviar estas recomendaciones, como por ejemplo la asignatura de Lengua Castellana y Literatura a través de actividades relacionadas sobre dicha temática (una redacción, un dictado, una exposición, etc.). Además, 
se debe hacer partícipe a las familias, debido a la enorme influencia que tienen sobre los hábitos de los adolescentes, pero también a las administraciones públicas, quienes deben apoyar en la medida de lo posible la implementación de programas de este tipo.

\section{Agradecimientos}

Dar las gracias a los Institutos de Educación Secundaria L’Almadrava y Mediterrània por su colaboración en el estudio, así como a los profesores que han permitido su realización durante la hora de Tutoría. Por supuesto, también quiero agradecer a los participantes su buena predisposición a la hora de formar parte de esta investigación.

\section{Referencias}

Aguilar, M. J., Ortegón, A., Mur, N., Sánchez, J. C., García, J. J., García, I., \& Sánchez, A. M. (2014). Programas de actividad física para reducir sobrepeso y obesidad en niños y adolescentes: revisión sistemática. Nutrición Hospitalaria, 30(4), 727-740.

Aibar, A., Julián, J.A., Murillo, B., García-González, L., Estrada, S., \& Bois, J. (2015). Actividad física y apoyo de la autonomía: El rol del profesor de Educación Física. Revista de Psicología del Deporte, 24(1), 155-161.

Ayechu, A., \& Durá, T. (2010). Calidad de los hábitos alimentarios (adherencia a la dieta mediterránea) en los alumnos de educación secundaria obligatoria. Anales del sistema sanitario de Navarra, 33(1), 35-42.

Beltrán, V. J., Sierra, A. C., Jiménez, A., González-Cutre, D., Martínez, C., \& Cervelló, E. (2017). Diferencias según género en el tiempo empleado por adolescentes en actividad sedentaria y actividad física en diferentes segmentos horarios del día. Retos. Nuevas tendencias en Educación Física, Deporte y Recreación, 31, 3-7.

Beltrán-Carrillo, V. J., \& Devís-Devís, J., \& Peiró-Velert, C. (2012). Actividad física y sedentarismo en adolescentes de la Comunidad Valenciana. Revista Internacional de Medicina y Ciencias de la Actividad Física y del Deporte, 12(45), 123-137.

Bibiloni, M. M., Fernández-Blanco, J., Pujol-Plana, N., Surià, S., Pujol-Puyané, M. C., Mercadé, S., ... \& Tur, J.A. (2017). Reversión de sobrepeso y obesidad en población infantil de Vilafranca del Penedès: Programa ACTIVA'T (2012). Gaceta Sanitaria. https://doi.org/10.1016/ j.gaceta.2017.10.002

Blakemore, S. J., \& Mills, K. L. (2014). Is adolescence a sensitive period for sociocultural processing? Annual Review of Psychology, 65, 187-207.

Bonfanti, N., Fernández, J. M., Gomez-Delgado, F., \& PérezJiménez, F. (2014). Efecto de dos dietas hipocalóricas y su combinación con ejercicio físico sobre la tasa metabólica basal y la composición corporal. Nutrición Hospitalaria, 29(3), 635-643.

Buckland, G, Bach, A., \& Serra, L. (2008). Eficacia de la dieta mediterránea en la prevención de la obesidad. Una revisión de la bibliografía. Revista Española de Obesidad, 6(6), 329-339.

Calatayud, F., Calatayud, B., \& Gallego, J. G. (2011). Efectos de una dieta mediterránea tradicional en niños con sobrepeso y obesidad tras un año de intervención. Revista Pediatría de Atención Primaria, 13(52), 553-569.

Chen, J. J., \& Lee, Y. (2013). Physical Activity for Health: Evidence, Theory and Practice. Journal of Preventive Medicine and Public Health, 46(Suppl 1), S1-S2.

Contreras, O. R., Fernández, J. G., García, L. M., Palou, P., \& Ponseti, J. (2010). El autoconcepto físico y su relación con la práctica deportiva en estudiantes adolescentes. Revista de Psicología del Deporte, 19(1), 23-39.

Crocker, P. R. E., Sabiston, C. M., Kowalski, K. C., McDonough, M. H., \& Kowalski, N. (2006). Longitudinal Assessment of the Relationship Between Physical SelfConcept and Health-Related Behavior and Emotion in Adolescent Girls. Journal of Applied Sport Psychology, 18(3), 185-200.

Cuervo, C., Cachón, J., González, C., \& Zagalaz, M. L. (2017). Hábitos alimentarios y práctica de actividad física en una muestra de adolescentes de una ciudad del norte de España. Journal of Sport and Health Research, 9(1), 7584.

De Gracia, M., Marcó, M., \& Trujado, P. (2007). Factores asociados a la conducta alimentaria en preadolescentes. Psicothema, 19(4), 646-653.

De la Torre, M. J., Ruiz-Ariza, A., López-Serrano, S., \& Martínez-López, E. J. (2018). La parentalidad transformacional se relaciona positivamente con el autoconcepto físico de jóvenes adolescentes. Retos. Nuevas tendencias en Educación Física, Deporte y Recreación, 34, 3-7.

Esnaola, I. (2005a). Autoconcepto físico y satisfacción corporal en mujeres adolescentes según el tipo de deporte practicado. Apunts. Educación Física y Deportes, 80, 512.

Esnaola, I. (2005b). Desarrollo del autoconcepto durante la adolescencia y principio de la juventud. Revista de Psicología General y Aplicada, 58(2), 265-277.

Esnaola, I., \& Revuelta, L. (2009). Relaciones entre la actividad física, autoconcepto físico, expectativas, valor percibido y dificultad percibida. Acción Psicológica, 6(2), 31-43.

Esnaola, I., Goñi, A., \& Madariaga, J. M. (2008). El autoconcepto: perspectivas de investigación. Revista de Psicodidáctica, 13(1), 179-194.

Esnaola, I., Rodríguez, A., \& Goñi, E. (2011). Propiedades psicométricas del cuestionario de Autoconcepto AF5. Anales de Psicología, 27(1), 109-117.

Esposito, K., Pontillo, A., Di Palo, C., Giugliano, G., Masella, M., Marfella, R., \& Giugliano, D. (2003). Effect of weight loss and lifestyle changes on vascular inflammatory markers in obese women: a randomized trial. Jama, 289(14), 1799-1804.

Estevan, I., Queralt, A., \& Molina García, J. (2018). Biking to school: the role of bicycle sharing programs in adolescents. Journal of School Health, 88(12), 871-876.

Estévez, M., Muros, J. J., Torres, B., Pradas, F., Zurita, F., \& Cepero, M. (2015). Influencia de la composición corporal y la aceptación por las clases de educación física sobre la autoestima de niños de 14-16 años de Alicante, España. Nutrición Hospitalaria, 31(4), 1519-1524. 
Fernández-Bustos, J. G., González-Martí, I., Contreras, O., \& Cuevas, R. (2015). Relación entre imagen corporal y autoconcepto físico en mujeres adolescentes. Revista Latinoamericana de Psicología, 47(1), 25-33.

Foster Schubert, K. E., Alfano, C. M., Duggan, C. R., Xiao, L., Campbell, K. L., Kong, A., ... \& McTiernan, A. (2012). Effect of diet and exercise, alone or combined, on weight and body composition in overweight to obese postmenopausal women. Obesity, 20(8), 1628-1638.

Fox, K. R., \& Corbin, C. B. (1989). The Physical Self-Perception Profile: Development and Preliminary Validation. Journal of Sport and Exercise Psychology, 11(4), 408-430.

Frimel, T. N., Sinacore, D. R., \& Villareal, D. T. (2008). Exercise attenuates the weight-loss-induced reduction in muscle mass in frail obese older adults. Medicine and Science in Sports and Exercise, 40(7), 1213-1219.

García-Reyna, N. I., Gussinyer, S., \& Carrascosa, A. (2007). Niñ@s en Movimiento, un programa para el tratamiento de la obesidad infantil. Medicina Clínica, 129(16), 619623.

González, G., Hernández, S., Pozo, P., \& García, D. (2011). Asociación entre tejido graso abdominal y riesgo de morbilidad: efectos positivos del ejercicio físico en la reducción de esta tendencia. Nutrición Hospitalaria, 26(4), 685-691.

Goñi, A., \& Rodríguez, A. (2004). Eating disorders, sport practice and physical self-concept in adolescents. Actas Españolas de Psiquiatría, 32(1), 29-36.

Goñi, A., \& Rodríguez, A. (2007). Variables associated with the risk for eating disorders in adolescence. Salud Mental, 30(4), 16-23.

Goñi, A., Ruiz de Azúa, S., \& Liberal, I. (2004). Las propiedades psicométricas de un nuevo cuestionario para la medida del autoconcepto físico. Revista de Psicología del Deporte, 13(2), 195-213.

Goñi, E., \& Infante, G. (2010). Actividad físico-deportiva, autoconcepto físico y satisfacción con la vida. European Journal of Education and Psychology, 3(2), 199-208.

Grao-Cruces, A., Nuviala, A., Fernández-Martínez, A., \& Martínez-López, E. J. (2015). Relationship of physical activity and sedentarism with tobacco and alcohol consumption, and Mediterranean diet in Spanish teenagers. Nutrición Hospitalaria, 31(4), 1693-1700.

Grao-Cruces, A., Nuviala, A., Fernández-Martínez, A., PorcelGálvez, A. M., Moral-García, J. E., \& Martínez-López, E. J. (2013). Adherencia a la dieta mediterránea en adolescentes rurales y urbanos del sur de España, satisfacción con la vida, antropometría y actividades físicas y sedentarias. Nutrición Hospitalaria, 28(3), 1129-1135.

Guillén, F., \& Ramírez, M. (2011). Relación entre el autoconcepto y la condición física en alumnos del Tercer Ciclo de Primaria. Revista de Psicología del Deporte, 20(1), 45-59.

Gussinyer, S., García-Reyna, N. I., Carrascosa, A., Gussinyer, M., Yeste, D., Clemente, M., \& Albisu, M. (2008). Cambios antropométricos, dietéticos y psicológicos tras la aplicación del programa «Niñ@s en movimiento»en la obesidad infantil. Medicina Clínica, 131(7), 245-249.

Gutiérrez, M. (2014). Relaciones entre el clima motivacional, las experiencias en educación física y la motivación in- trínseca de los alumnos. Retos. Nuevas tendencias en Educación Física, Deporte y Recreación, 26, 9-14.

Kahlmeier, S., Wijnhoven, T. M., Alpiger, P., Schweizer, C., Breda, J., \& Martin, B. W. (2015). National physical activity recommendations: systematic overview and analysis of the situation in European countries. BMC Public Health, 15, 133.

Keys, A., Menotti, A., Karvonen, M. J., Aravanis, C., Blackburn, H., Buzina, R., ... \& Toshima, H. (1986). The diet and 15-year death rate in the seven countries study. American Journal of Epidemiology, 124(6), 903915.

Lamoneda, J., \& Huertas, F. J. (2017). Análisis de la práctica deportiva-recreativa a través de un programa de promoción en el recreo en función del sexo en adolescentes españoles. Retos. Nuevas tendencias en Educación Física, Deporte y Recreación, 32, 25-29.

Loland, N, W. (1998). Body image and physical activity. A survey among Norwegian men and women. International Journal of Sport and Exercise Psychology, 29(4), 339365.

Mariscal-Arcas, M., Rivas, A., Velasco, J., Ortega, M., Caballero, A. M., \& Olea-Serrano, F. (2009). Evaluation of the Mediterranean Diet Quality Index (KIDMED) in children and adolescents in Southern Spain. Public Health Nutrition, 12(9), 1408-1412.

Marques, A., González, M., Martins, J., Fernández-Villarino, M. A., \& Carreiro, F. (2017). Relación entre la actividad física de los adolescentes y la de madres/padres. Revista de Psicología del Deporte, 26(1), 145-156.

Menotti, A., Kromhout, D., Blackburn, H., Fidanza, F., Buzina, R., \& Nissinen, A. (1999). Food intake patterns and 25year mortality from coronary heart disease: cross-cultural correlations in the Seven Countries Study. European Journal of Epidemiology, 15(6), 507-515.

Molero, D., Ortega, F., Valiente, I., \& Zagalaz, M. L. (2010). Estudio comparativo del autoconcepto físico en adolescentes en función del género y del nivel de actividad físico-deportiva. Retos. Nuevas tendencias en Educación Física, Deporte y Recreación, 17, 38-41.

Moreno, J. A., \& Cervelló, E. (2004). Influencia de la actitud del profesor en el pensamiento del alumno hacia la Educación Física. Revista Internacional de Ciencias Sociales y Humanidades, 14(1), 33-51.

Moreno, J. A., \& Cervelló, E. (2005). Physical self-perception in Spanish adolescents: effects of gender and involvement in physical activity. Journal of Human Movement Studies, 48(4), 291-311.

Moreno, J.A., Cervelló, E., \& Moreno, R. (2008). Importancia de la práctica físico-deportiva y del género en el autoconcepto físico de los 9 a los 23 años. International Journal of Clinical and Health Psychology, 8(1), 171183.

Moreno, J. A., Moreno, R., \& Cervelló, E. (2007). El autoconcepto físico como predictor de la intención de ser físicamente activo. Psicología y Salud, 17(2), 261267.

Moreno, J. A., Moreno, R., \& Cervelló, E. (2009). Relación del autoconcepto físico con las conductas de consumo de alcohol y tabaco en adolescentes. Adicciones, 21(2), 147- 
154.

Morton, K. L., Wilson, A. H., Perlmutter, L. S., \& Beauchamp, M. R. (2012). Family leadership styles and adolescent dietary and physical activity behaviors: a cross-sectional study. International Journal of Behavioral Nutrition and Physical Activity, 9(1), 48-56.

Organización Mundial de la Salud. (2010). Recomendaciones Mundiales sobre Actividad Física para la Salud. Disponible en http://www.who.int/dietphysicalactivity/ factsheet_recommendations/es/

Oviedo, G., Sánchez, J., Castro, R., Calvo, M., Sevilla, J. C., Iglesias, A., \& Guerra, M. (2013). Niveles de actividad física en población adolescente: estudio de caso. Retos. Nuevas tendencias en Educación Física, Deporte y Recreación, 23, 43-47.

Physical Activity Guidelines Advisory Committee. (2018). Physical activity guidelines advisory committee scientific report. Washington, DC: US Department of Health and Human Services.

Piéron, M. \& Ruiz-Juan, F. (2013). Influencia del ámbito familiar e iguales en los hábitos físico-deportivos de los jóvenes. Revista Internacional de Medicina y Ciencias de la Actividad Física y el Deporte, 13(51), 525-549.

Ramos, P., Jiménez-Iglesias,A., Rivera, F., \& Moreno, C. (2016). Evolución de la práctica de la actividad física en los adolescentes españoles. Revista Internacional de Medicina y Ciencias de la Actividad Física y del Deporte, 16(62), 335-353.

Reigal, R. E., \& Videra, A. (2011). Frecuencia de práctica física y autoconcepto físico multidimensional en la adolescencia. Apunts. Educación Física y Deporte, 105, 28-34.

Revuelta, L., \& Esnaola, I. (2011). Clima familiar deportivo y autoconcepto físico en la adolescencia. European Journal of Education and Psychology, 4(1), 19-31.

Revuelta, L., Esnaola, I., \& Goñi, A. (2013). El autoconcepto físico como determinante de la actividad físico-deportiva durante la adolescencia. Psicología Conductual, 21(3), 581-601.

Revuelta, L., Esnaola, I., \& Goñi, A. (2016). Relaciones entre el autoconcepto físico y la actividad físico-deportiva adolescente. Revista Internacional de Medicina y Ciencias de la Actividad Física y del Deporte, 16(63), 561581.

Rodríguez, A., Goñi, A., \& Ruiz de Azúa, S. (2006). Autoconcepto físico y estilos de vida en la adolescencia. Psychosocial Intervention, 15(1), 81-94.

Rosa, A., Carrillo, P. J., García, E., Pérez, J. J., Tarraga, L., \&
Tarra, P. J. (2018). Dieta mediterránea, estado de peso y actividad física en escolares de la Región de Murcia. Clínica e Investigación en Arteriosclerosis. https://doi.org/10.1016/j.arteri.2018.09.002

Serra-Majem, L., Ribas, L., Ngo, J., Ortega, R. M., García, A., Pérez-Rodrigo, C., \& Aranceta, J. (2004). Food, youth and the Mediterranean diet in Spain. Development of KIDMED, Mediterranean Diet Quality Index in children and adolescents. Public Health Nutrition, 7(7), 931-935.

Sevil, J., Abós, Á., Generelo, E., Aibar, A., \& García-González, L. (2016). Importancia del apoyo a las necesidades psicológicas básicas en la predisposición hacia diferentes contenidos en Educación Física. Retos. Nuevas tendencias en Educación Física, Deporte y Recreación, 29, 3-8.

Shavelson, R. J., Hubner, J. J., \& Stanton, J. C. (1976). Selfconcept: validation of construct interpretations. Review of Educational Research, 46(3), 407-441.

Slutzky, C. B., \& Simpkins, S. D. (2009). The link between children's sport participation and self-esteem: exploring the mediating role of sport self-concept. Psychology of Sport and Exercise, 10(3), 381-389.

Stephens, S. K., Cobiac, L. J., \& Veerman, J. L. (2014). Improving diet and physical activity to reduce population prevalence of overweight and obesity: An overview of current evidence. Preventive Medicine, 62, 167-178.

Tunstall-Pedoe, H., Kuulasmaa, K., Mähönen, M., Tolonen, H., Ruokokoski, E., \& Amouyel, P. (1999). Contribution of trends in survival and coronar y-event rates to changes in coronary heart disease mortality: 10-year results from 37 WHO MONICA Project populations. The Lancet, 353(9164), 1547-1557.

Usán, P., Salavera, C., Murillo, V., \& Merino, A. (2018). Relación conductual de la motivación y el autoconcepto físico en el consumo de drogas de adolescentes deportistas. Retos. Nuevas tendencias en Educación Física, Deporte y Recreación, 33, 40-45.

Videra-García, A., \& Reigal-Garrido, R. (2013). Autoconcepto físico, percepción de salud y satisfacción vital en una muestra de adolescentes. Anales de Psicología, 29(1), 141-147.

Warburton, D. E., Nicol, C. W., \& Bredin, S. S. (2006). Health benefits of physical activity: the evidence. Canadian Medical Association Journal, 174(6), 801-809.

Willett, W. C., Sacks, F., Trichopoulou, A., Drescher, G., Ferro-Luzzi, A., Helsing, E., \& Trichopoulous, D. (1995). Mediterranean diet pyramid: a cultural model for healthy eating. The American Journal of Clinical Nutrition, 61(Suppl 6), 1402S-1406S.

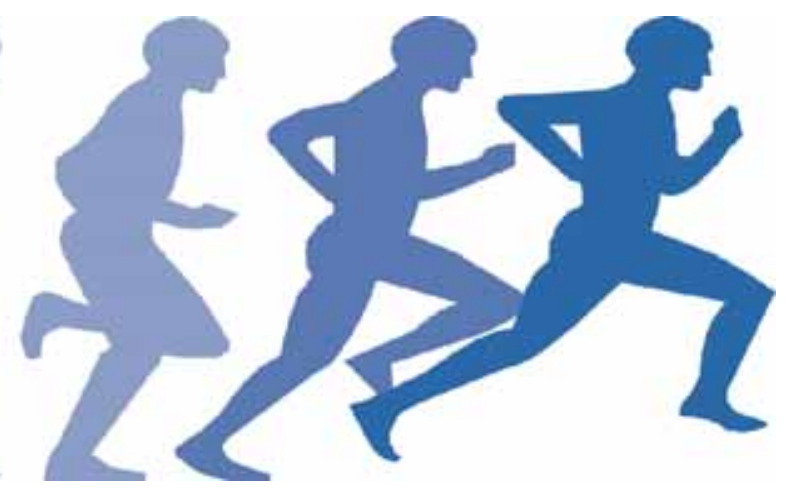

